Research Article

www.ijrap.net

\title{
FOLK REMEDIES OF MEDICINAL PLANTS FOR SNAKE BITES, SCORPION STINGS AND DOG BITES IN EASTERN GHATS OF KOLLI HILLS, TAMIL NADU, INDIA
}

Prabu M* and Kumuthakalavalli R

Department of Biology, Gandhigram Rural Institute-Deemed University, Gandhigram-624 302 Tamil Nadu India

Received on: 08/06/12 Revised on: 06/07/12 Accepted on: 09/09/12

\author{
*Corresponding author \\ Email: sma.subi@gmail.com \\ DOI: $10.7897 / 2277-4343.03523$ \\ Published by Moksha Publishing House. Website www.mokshaph.com \\ All rights reserved.
}

\begin{abstract}
Folk remedies practiced by the tribes of Kolli Hills of Eastern Ghats, Tamil Nadu, India were documented. Around 30 folk remedies for snake bite, 18 for scorpion sting and 4 for dog bite were reported in this study. Details of the plants used for snake bite, scorpion sting and dog bite with local name, parts used and methods of preparation, dosage and the mode of administration were discussed.

Keywords: Folk remedies, snake bite, scorpion sting, dog bite, Eastern Ghats.
\end{abstract}

\section{INTRODUCTION}

Plants have been used in traditional medicine for thousands of years. The knowledge of medicinal plants has been accumulated from different medicinal systems such as Ayurveda, Unani and Siddha. In India, it is reported that traditional healers use 2,500 plant species and 100 species of plants serve as regular source of medicine. ${ }^{1}$ In the last few decades there has been an increasing interest in the study of medicinal plants and their traditional use in different parts of the world. ${ }^{2}$ In developed countries, 25 per cent of the medicinal drugs used are from plants and their derivatives. ${ }^{3}$ Traditional medicinal knowledge is important not only for its potential contribution to drug development and market values but also for the healthcare professionals. Documenting the indigenous knowledge through ethnobotanical studies is important for conservation and utilization of biological resources. Tribal groups, forest dwellers and rural people possess unique knowledge about plants and their uses. ${ }^{4}$ According to $\mathrm{WHO}^{5} 80$ per cent of the world population depend upon traditional medicine for their primary healthcare needs. Several medicinal plants with accepted therapeutics in the healthcare are now attracting greater attention for the fact that they are shedding more light on their hidden biologically active principles. ${ }^{6}$

Although many studies concerning the ethnobotanical studies of common ailments such as cuts, wounds, cold, cough, fever, asthma, chronic disorders, ulcer, leprosy, rheumatism, antidotes in poisonous bites and veterinary diseases have been carried out targeting the Kolli Hills, ${ }^{7}$ there is scarce information on the folk remedies for snake bite, scorpion sting and dog bite. ${ }^{8}$

Besides this, it is made known that the traditional healers are dwindling in number; the younger generation is not interested to carry on this tradition of folklore remedies. Hence there is a grave danger of disappearance of traditional knowledge. Therefore, it becomes the responsibility of the scientific community to unravel the information and to document the folklore remedies for availability to the whole world for the benefit of human beings. Therefore the present study was undertaken to document the detailed information on medicinal plants used by tribes and traditional healers in Kolli Hills of Eastern Ghats for snake bite, scorpion sting and dog bite.

\section{MATERIALS AND METHODS}

Eastern Ghats of India is ethnobotanically very rich in having a wide variety of medicinal plants. ${ }^{9}$ With its diverse topographical condition; this region is well suited for a range of medicinal plant species. Eastern Ghats of Kolli Hills is situated in the Namakkal District of Tamil $\mathrm{Nadu}$ with an area of 441.8 sq. $\mathrm{km}$. The area of investigation (Figure 1) lies between $11^{\circ} 10^{\prime}-11^{\circ} 30^{\prime} \mathrm{N}$ latitude $75^{\circ} 15^{\prime}-75^{\circ} 30^{\prime} \mathrm{E}$ longitude. The hill is bounded by Namakkal in the South and South West, Rasipuram in the North West, Attur in the North East and Tiruchirapalli in the South East region and this is attributed to the richness of the flora and several localized forests. ${ }^{10}$ Kolli Hills on the western, eastern and southern sides rise abruptly from the plains and on the northern side ascend to the plains by numerous long and gently sloping spurs. As per 2011 census, the total population of Kolli Hills is about 42,200 in 14 villages. The present study on ethnobotanical survey of medicinal plants used for snake bite, scorpion sting and dog bite was carried out at four different areas such as Semmedu, Solakkadu, Ariyur Solakkadu and Keel Solakkadu of Kolli Hills.

Data collection on ethnobotanical knowledge was carried out on 2010-2011 using a specific questionnaire. ${ }^{11}$ The plants were collected with the help of traditional healers and information on the medicinal plants such as vernacular name, parts used, time of collection of the plant material, the methods of preparation of botanical products, dosage and mode of administration of various herbal remedies were recorded. The information recorded was further crosschecked by consulting the beneficiaries, villagers and also two traditional healers who are above 60 years old and who are in the occupation for more than 30 years. The medicinal plants were botanically identified 
using the Flora of Tamil Nadu Carnatic ${ }^{12}$ and an Excursion Flora of Central Tamil Nadu. ${ }^{13}$ The herbarium specimens were deposited in the Department of Biology, Gandhigram Rural Institute, Gandhigram, for the future reference.

\section{RESULTS AND DISCUSSION}

Generally each and every plant has medicinal value. In the present study 35 plant species of medicinal plants belonging to 34 genera of 23 families for the snake bite; 28 plant species belonging to 27 genera of 21 families for scorpion sting and 12 plant species of 12 genera of 11 families to cure dog bite are reported. Totally about 52 plant species of 29 families distributed in 49 genera are reported to treat snake bite, scorpion sting and dog bite. The detailed information such as the local name of the plant, parts used and methods of preparation of botanical products, dosage and mode of administration were documented from the local people, tribes and traditional healers of Kolli Hills in Namakkal District of Tamil $\mathrm{Nadu}$. The medicinal plants are being taken orally either raw or in the form of crude extracts, juice, decoction, infusion, paste, powder, tincture, ash and also externally as oil (Table 1).

\begin{tabular}{|c|c|c|c|c|c|c|c|c|}
\hline \multirow[t]{2}{*}{ Botanical Name } & \multirow{2}{*}{$\begin{array}{l}\text { Vernacular } \\
\text { Name }\end{array}$} & \multirow[t]{2}{*}{ Family } & \multirow{2}{*}{$\begin{array}{l}\text { Useful } \\
\text { Parts }\end{array}$} & \multicolumn{3}{|c|}{ Uses } & \multirow[t]{2}{*}{ Other Medicinal Uses } & \multirow{2}{*}{$\begin{array}{l}\text { Preparations } \\
\text { \& Mode of } \\
\text { Application } \\
\end{array}$} \\
\hline & & & & S.B & S.S & D.B & & \\
\hline $\begin{array}{l}\text { Abrus precatorius } \\
\text { L. }\end{array}$ & Kundumani & Fabaceae & $\begin{array}{l}\text { Roots, } \\
\text { Leaves }\end{array}$ & $\sqrt{ }$ & & & $\begin{array}{l}\text { Nervous disorders in } \\
\text { children, cuts, wounds, } \\
\text { inflammation. }\end{array}$ & $\begin{array}{l}\text { Paste (internal } \\
\& \text { external) }\end{array}$ \\
\hline $\begin{array}{l}\text { Achyranthes } \\
\text { aspera L. }\end{array}$ & Naaiuruvi & Amaranthaceae & $\begin{array}{c}\begin{array}{c}\text { Whole } \\
\text { plant }\end{array} \\
\end{array}$ & $\sqrt{ }$ & & $\sqrt{ }$ & $\begin{array}{l}\text { Headache, to increase } \\
\text { breast milk. }\end{array}$ & $\begin{array}{l}\text { Decoction } \\
\text { (internal) }\end{array}$ \\
\hline Aloe vera $\mathrm{L}$. & Kathalai & Liliaceae & $\begin{array}{l}\text { Whole } \\
\text { plant }\end{array}$ & & $\sqrt{ }$ & & $\begin{array}{c}\text { Kidney stones, ulcer, cold, } \\
\text { ear problems, typhoid, } \\
\text { malaria. }\end{array}$ & $\begin{array}{l}\text { Juice (internal } \\
\& \text { external) }\end{array}$ \\
\hline $\begin{array}{c}\text { Anisomeles } \\
\text { malabarica } \text { R.Br. }\end{array}$ & Peimiratti & Lamiaceae & $\begin{array}{l}\text { Whole } \\
\text { plant }\end{array}$ & $\sqrt{ }$ & $\sqrt{ }$ & & $\begin{array}{c}\text { Stomach \& bowel problem, } \\
\text { to expel gas from } \\
\text { intestines. }\end{array}$ & Paste (internal) \\
\hline $\begin{array}{l}\text { Aristolochia indica } \\
\text { L. }\end{array}$ & Urikakodi & $\begin{array}{c}\text { Aristolochiacea } \\
\text { e }\end{array}$ & $\begin{array}{c}\text { Whole } \\
\text { plant }\end{array}$ & $\sqrt{ }$ & $\sqrt{ }$ & & $\begin{array}{c}\text { Wounds, fever, emesis, } \\
\text { nausea. }\end{array}$ & Paste (external) \\
\hline $\begin{array}{l}\text { Azadirachta indica } \\
\text { A. Juss. }\end{array}$ & Vembu & Meliaceae & Flowers & $\sqrt{ }$ & & & $\begin{array}{l}\text { Stomach ache, intestinal } \\
\text { worms, ulcer, malaria, } \\
\text { fever, eczema. }\end{array}$ & Juice (internal) \\
\hline $\begin{array}{c}\text { Blepharis } \\
\text { maderaspatensis } \\
\text { L. }\end{array}$ & Naikalli & Acanthaceae & Leaves & $\sqrt{ }$ & $\sqrt{ }$ & $\sqrt{ }$ & $\begin{array}{l}\text { Venereal diseases, malaria } \\
\text { and jaundice. }\end{array}$ & Juice (internal) \\
\hline $\begin{array}{l}\text { Calendula } \\
\text { officinalis L. }\end{array}$ & Marikollundu & Asteraceae & Flowers & $\sqrt{ }$ & & & $\begin{array}{l}\text { Incised wounds, burns, } \\
\text { ulcers, skin diseases. }\end{array}$ & Juice (internal) \\
\hline $\begin{array}{c}\text { Calotropis } \\
\text { gigantea } \mathrm{L} . \mathrm{R} . \mathrm{Br} .\end{array}$ & Erukku & Asclepiadiaceae & Roots & $\sqrt{ }$ & & & $\begin{array}{c}\text { Jaundice, fever, eye } \\
\text { diseases, male fertility } \\
\text { disorders, cuts, wounds. }\end{array}$ & $\begin{array}{l}\text { Paste (internal } \\
\& \text { external) }\end{array}$ \\
\hline $\begin{array}{l}\text { Cardiospermum } \\
\text { helicacabum } \mathrm{L} .\end{array}$ & Mudakothan & Sapindaceae & Leaves & $\sqrt{ }$ & & & $\begin{array}{l}\text { Rheumatic pain, swellings, } \\
\text { tumours, rheumatoid } \\
\text { arthritis }\end{array}$ & Paste (external) \\
\hline Cassia alata $\mathrm{L}$. & Semaiagatti & Caesalpiniaceae & Leaves & $\sqrt{ }$ & $\sqrt{ }$ & $\sqrt{ }$ & $\begin{array}{l}\text { Vaginal discharges, } \\
\text { ringworm, cancer, } \\
\text { paralysis, rheumatism. }\end{array}$ & Paste (internal) \\
\hline Cassia tora $\mathrm{L}$. & Tagarai & Caesalpiniaceae & Seeds & $\sqrt{ }$ & & & $\begin{array}{l}\text { Leucorrhoea, fever, } \\
\text { asthma, cough, ulcer, } \\
\text { wounds, chronic } \\
\text { constipation. }\end{array}$ & $\begin{array}{l}\text { Decoction } \\
\text { (internal) }\end{array}$ \\
\hline $\begin{array}{c}\text { Cissus } \\
\text { quadrangularis } \mathrm{L} .\end{array}$ & Pirandai & Vitaceae & $\begin{array}{l}\text { Seeds, } \\
\text { Leaves }\end{array}$ & & $\sqrt{ }$ & & $\begin{array}{c}\text { Tumour, malarial fever, } \\
\text { diarrhoea, caused by } \\
\text { indigestion, easy delivery. }\end{array}$ & $\begin{array}{l}\text { Juice (internal) } \\
\text { Paste (external) }\end{array}$ \\
\hline Citrus limon L. & Elumichai & Rutaceae & Fruits & $\sqrt{ }$ & & & $\begin{array}{l}\text { Dry skin, making body } \\
\text { color fairer, eczema, } \\
\text { furuncle, pimples. }\end{array}$ & Paste (external) \\
\hline $\begin{array}{c}\text { Curcuma } \\
\text { angustifolia } \text { Roxb. }\end{array}$ & Kattumanchal & Zingiberaceae & $\begin{array}{c}\text { Rhizom } \\
\mathrm{e}\end{array}$ & $\sqrt{ }$ & $\sqrt{ }$ & $\sqrt{ }$ & $\begin{array}{l}\text { Wounds, cuts, body ache, } \\
\text { bone fracture, dysentery, } \\
\text { swellings. }\end{array}$ & Paste (external) \\
\hline Curcuma longa $\mathrm{L}$. & Manchal & Zingiberaceae & $\begin{array}{c}\text { Rhizom } \\
\text { e }\end{array}$ & & $\sqrt{ }$ & & $\begin{array}{l}\text { Ringworm, scabies, cough, } \\
\text { bronchitis, indigestion, } \\
\text { cuts, wounds. }\end{array}$ & Paste (external) \\
\hline $\begin{array}{l}\text { Cyperus rotundus } \\
\text { L. }\end{array}$ & Koraipul & Cyperaceae & Roots & $\sqrt{ }$ & & & $\begin{array}{l}\text { Microbial contaminations, } \\
\text { body pain, malaria, } \\
\text { diarrhoea, dysentery. }\end{array}$ & Paste (external) \\
\hline Datura metel L. & Oomathai & Solanaceae & Roots & $\sqrt{ }$ & & & $\begin{array}{l}\text { Male fertility disorders, } \\
\text { partial paralysis, mental } \\
\text { disorders. }\end{array}$ & Paste (internal) \\
\hline $\begin{array}{l}\text { Dodonea viscosa } \\
\text { L. Jacq. }\end{array}$ & Velari & Sapindaceae & Leaves & $\sqrt{ }$ & & & $\begin{array}{c}\text { Intestinal worms, fish } \\
\text { poison, swellings, } \\
\text { rheumatic joints, jaundice. }\end{array}$ & Paste (external) \\
\hline Eclipta alba $\mathrm{L}$. & Karisilanganni & Asteraceae & Leaves & $\sqrt{ }$ & $\sqrt{ }$ & $\sqrt{ }$ & $\begin{array}{l}\text { Injury caused due to } \\
\text { continuous contact with }\end{array}$ & Juice (internal) \\
\hline
\end{tabular}


Prabu M et al / IJRAP 3(5), Sep - Oct 2012

\begin{tabular}{|c|c|c|c|c|c|c|c|c|}
\hline & & & & & & & mud. & \\
\hline $\begin{array}{c}\text { Eclipta prostrata } \\
\text { L. }\end{array}$ & $\begin{array}{c}\text { Manchal } \\
\text { Karisilanganni }\end{array}$ & Asteraceae & Leaves & $\sqrt{ }$ & $\sqrt{ }$ & & $\begin{array}{c}\text { Catarrh, malarial fever, } \\
\text { body inflammation, } \\
\text { jaundice. }\end{array}$ & Paste (external) \\
\hline $\begin{array}{c}\text { Gloriosa superba } \\
\text { L. }\end{array}$ & $\begin{array}{l}\text { Kalapaih } \\
\text { Kilangu }\end{array}$ & Liliaceae & Tuber & $\sqrt{ }$ & $\sqrt{ }$ & $\sqrt{ }$ & $\begin{array}{l}\text { Quite delivery, Vaginal } \\
\text { uterine prolapsed, piles, } \\
\text { ulcers, leprosy. }\end{array}$ & Paste (external) \\
\hline $\begin{array}{c}\text { Gymnema } \\
\text { sylvestre } \mathrm{R} . \mathrm{Br} .\end{array}$ & Sirukurinchaan & Asclepiadaceae & Roots & $\sqrt{ }$ & & & $\begin{array}{c}\text { Wounds, diabetes, eye } \\
\text { problems, fever. }\end{array}$ & Juice (internal) \\
\hline $\begin{array}{l}\text { Helianthus annus } \\
\text { L. }\end{array}$ & Suriyakanthi & Asteraceae & Seeds & & $\sqrt{ }$ & & $\begin{array}{l}\text { Intermittent fever, body } \\
\text { cool, nasal haemorrhage. }\end{array}$ & Oil (external) \\
\hline $\begin{array}{c}\text { Hemidesmus } \\
\text { indicus } \text { L.Schultes. }\end{array}$ & Nantamul & Asclepiadiaceae & Roots & $\sqrt{ }$ & & & $\begin{array}{l}\text { Inflammation, diabetes, } \\
\text { fever, hair growth, ulcers, } \\
\text { rheumatism. }\end{array}$ & $\begin{array}{l}\text { Decoction } \\
\text { (internal) }\end{array}$ \\
\hline $\begin{array}{c}\text { Hybanthus } \\
\text { enneaspermus L.F. }\end{array}$ & Orilaithamatai & Violaceae & $\begin{array}{l}\text { Whole } \\
\text { plant }\end{array}$ & & $\sqrt{1}$ & & $\begin{array}{l}\text { As diuretic, as demulcent, } \\
\text { bowel complaints in } \\
\text { children. }\end{array}$ & Juice (internal) \\
\hline $\begin{array}{l}\text { Kalanchoe pinnata } \\
\text { (Forsk.) Pers. }\end{array}$ & Katakataka & Crassulaceae & Leaves & & $\sqrt{ }$ & & $\begin{array}{l}\text { Venomous insect bite, } \\
\text { rheumatism, stiff joints, } \\
\text { kidney stones. }\end{array}$ & Paste (external) \\
\hline $\begin{array}{l}\text { Leucas aspera } \\
\text { (Wild.) Link. }\end{array}$ & Thumbai & Lamiaceae & Leaves & $\sqrt{ }$ & $\sqrt{ }$ & & $\begin{array}{c}\text { Tooth ache, chronic } \\
\text { asthma, eye problems, } \\
\text { fever, head ache. }\end{array}$ & $\begin{array}{l}\text { Decoction } \\
\text { (internal) }\end{array}$ \\
\hline $\begin{array}{c}\text { Mangifera indica } \\
\text { L. }\end{array}$ & Mamaram & Anacardiaceae & Petiole & & $\sqrt{ }$ & & $\begin{array}{l}\text { Diabetes, removal of tooth, } \\
\text { diarrhoea, throat infection, } \\
\text { wounds. }\end{array}$ & Paste (internal) \\
\hline $\begin{array}{c}\text { Michelia } \\
\text { champaga } \mathrm{L} .\end{array}$ & Champagam & Magnoliaceae & Leaves & & $\sqrt{ }$ & & $\begin{array}{l}\text { Indolent swellings, body } \\
\text { cool, cuts, wounds, skin } \\
\text { diseases. }\end{array}$ & Juice (internal) \\
\hline Mimosa pudica L. & Thottal surungi & Mimosaceae & $\begin{array}{l}\text { Whole } \\
\text { plant }\end{array}$ & $\sqrt{1}$ & $\sqrt{ }$ & & $\begin{array}{l}\text { Diabetes, boils, scabies, } \\
\text { cuts, wounds, } \\
\text { inflammation. }\end{array}$ & Paste (external) \\
\hline $\begin{array}{l}\text { Moringa oleifera } \\
\text { Lam. }\end{array}$ & Murungaih & Moringaceae & $\begin{array}{l}\text { Root } \\
\text { bark }\end{array}$ & $\sqrt{ }$ & & $\sqrt{ }$ & $\begin{array}{l}\text { Diarrhoea, abscess on eyes, } \\
\text { earache, stomach ache, cuts } \\
\text { and wounds. }\end{array}$ & Paste (external) \\
\hline $\begin{array}{l}\text { Mucuna pruriens } \\
\text { (L.) DC. }\end{array}$ & Poonaikkali & Fabaceae & $\begin{array}{l}\text { Seeds, } \\
\text { Whole } \\
\text { plant }\end{array}$ & $\sqrt{ }$ & $\sqrt{ }$ & & $\begin{array}{c}\text { Asthma, cancer, mumps, } \\
\text { diarrhoea, ringworm, } \\
\text { syphilis. }\end{array}$ & $\begin{array}{l}\text { Decoction \& } \\
\text { powder } \\
\text { (internal \& } \\
\text { external) } \\
\end{array}$ \\
\hline $\begin{array}{c}\text { Murraya Koeinegii } \\
\text { L. }\end{array}$ & Karuveppilai & Rutaceae & $\begin{array}{l}\text { Leaves, } \\
\text { bark, } \\
\text { root } \\
\text { bark } \\
\end{array}$ & $\sqrt{ }$ & $\sqrt{ }$ & $\sqrt{ }$ & $\begin{array}{l}\text { Toothache, dysentery, } \\
\text { diarrhoea, to check } \\
\text { vomiting. }\end{array}$ & $\begin{array}{l}\text { Juice (internal } \\
\& \text { external) }\end{array}$ \\
\hline $\begin{array}{c}\text { Musa paradisiaca } \\
\text { L. }\end{array}$ & Valaimaram & Musaeeae & Bark & $\sqrt{ }$ & $\sqrt{ }$ & $\sqrt{ }$ & Leprosy, mental disorders. & Juice (internal) \\
\hline $\begin{array}{l}\text { Nerium oleander } \\
\text { L. }\end{array}$ & Arali & Apocyanaceae & Seeds & $\sqrt{ }$ & & & $\begin{array}{l}\text { Heart problems, paralysis, } \\
\text { skin diseases. }\end{array}$ & Paste (external) \\
\hline $\begin{array}{l}\text { Ocimum sanctum } \\
\text { L. }\end{array}$ & Tulsi & Lamiaceae & Leaves & $\sqrt{ }$ & & & $\begin{array}{l}\text { Cancer, arthritis, oedema, } \\
\text { venereal diseases, male } \\
\text { fertility disorders, diabetes. }\end{array}$ & Juice (internal) \\
\hline $\begin{array}{l}\text { Pergularia daemia } \\
\text { Forsk. } \\
\end{array}$ & Veliparuthi & Asclepiadiaceae & Leaves & $\sqrt{ }$ & $\sqrt{ }$ & & $\begin{array}{c}\text { Skin diseases, cuts, } \\
\text { wounds, bronchial asthma. }\end{array}$ & Juice (internal) \\
\hline $\begin{array}{l}\text { Phyllanthus } \\
\text { emblica } \mathrm{L} .\end{array}$ & Nelli & Euphorbiaceae & Flowers & & & $\sqrt{ }$ & $\begin{array}{c}\text { Scabies, sores, nostril } \\
\text { haemorrhage, leucorrhoea, } \\
\text { gonorrhoea, syphilis. }\end{array}$ & Juice (internal) \\
\hline Piper nigrum L. & Milagu & Piperaceae & $\begin{array}{l}\text { Floral } \\
\text { buds }\end{array}$ & $\sqrt{ }$ & & & $\begin{array}{l}\text { Leprosy, skin diseases, } \\
\text { cuts, burns, cough, cold, } \\
\text { mouth ulcer. }\end{array}$ & Paste (internal) \\
\hline $\begin{array}{c}\text { Punica granatum } \\
\text { L. }\end{array}$ & Madhulai & Punicaceae & $\begin{array}{l}\text { Whole } \\
\text { plant }\end{array}$ & $\sqrt{ }$ & & & $\begin{array}{l}\text { Scabies, salivation with } \\
\text { nausea, antifertility, skin } \\
\text { diseases. }\end{array}$ & Paste (external) \\
\hline $\begin{array}{c}\text { Rauvolfia } \\
\text { serpentina } \mathrm{L} . \\
\text { Benth. Exkurz. }\end{array}$ & Sarpagandha & Apocynaceae & Root & $\sqrt{ }$ & & & $\begin{array}{l}\text { Stomach pain, to eliminate } \\
\text { intestinal worms, to reduce } \\
\text { blood pressure. }\end{array}$ & Paste (external) \\
\hline $\begin{array}{l}\text { Rhinachanthus } \\
\text { nasutus L. }\end{array}$ & Nagamalli & Acanthaceae & Leaves & $\sqrt{ }$ & & & $\begin{array}{c}\text { Domesticated animal } \\
\text { diseases, cuts. }\end{array}$ & Juice (internal) \\
\hline $\begin{array}{c}\text { Ricinus communis } \\
\text { L. }\end{array}$ & Aamanakku & Euphorbiaceae & Seeds & & $\sqrt{ }$ & $\sqrt{ }$ & $\begin{array}{l}\text { Diabetes, rheumatic fever, } \\
\text { malaria, typhoid, jaundice, } \\
\text { enhances fertility. }\end{array}$ & $\begin{array}{l}\text { Infusion } \\
\text { (external) }\end{array}$ \\
\hline $\begin{array}{c}\text { Solanum } \\
\text { virginianum } \mathrm{L} \text {. }\end{array}$ & Kandangkattiri & Solanaceae & Flower & & $\sqrt{ }$ & & $\begin{array}{c}\text { Emesis, diabetes, } \\
\text { diarrhoea, skin diseases, } \\
\text { venereal diseases, ulcer. }\end{array}$ & $\begin{array}{l}\text { Paste (internal } \\
\text { and external) }\end{array}$ \\
\hline $\begin{array}{c}\text { Tephrosia } \\
\text { purpurea } \text { L. Pers. }\end{array}$ & Kolunchi & Fabaceae & Root & & $\sqrt{ }$ & & $\begin{array}{l}\text { As astringent, obstruction } \\
\text { of spleen, kidneys, } \\
\text { pulmonary disease. }\end{array}$ & $\begin{array}{l}\text { Decoction } \\
\text { (internal) }\end{array}$ \\
\hline
\end{tabular}


Prabu M et al / IJRAP 3(5), Sep - Oct 2012

\begin{tabular}{|c|c|c|c|c|c|c|c|}
\hline $\begin{array}{l}\text { Terminalia arjuna } \\
\text { Wight \& Arn. }\end{array}$ & Marutham & Combretaceae & Wood & & $\sqrt{ }$ & $\begin{array}{l}\text { Jaundice, malaria, } \\
\text { dysentery, heart diseases, } \\
\text { leprosy, liver complaints. }\end{array}$ & Ash (internal) \\
\hline $\begin{array}{c}\text { Tragia involucrata } \\
\text { L. }\end{array}$ & Naipullu & Euphorbiaceae & $\begin{array}{l}\text { Whole } \\
\text { plant }\end{array}$ & $\sqrt{ }$ & & $\begin{array}{l}\text { Local swelling of hand and } \\
\text { feet, headache, fever. }\end{array}$ & Juice (internal) \\
\hline $\begin{array}{c}\text { Tribulus terrestris } \\
\text { L. }\end{array}$ & Nerinji & Zygophyllaceae & Flowers & & $\sqrt{ }$ & $\begin{array}{c}\text { Urinary troubles, heart } \\
\text { diseases, blood } \\
\text { purification. }\end{array}$ & Juice (internal) \\
\hline $\begin{array}{c}\text { Vernonia cinerea } \\
\text { Less. }\end{array}$ & Mukkuttipuntu & Asteraceae & $\begin{array}{l}\text { Whole } \\
\text { plant }\end{array}$ & & $\sqrt{ }$ & $\begin{array}{l}\text { Skin diseases, constipation, } \\
\text { fever, rheumatism, } \\
\text { conjunctives. }\end{array}$ & Juice (internal) \\
\hline Vitex trifolia L.F. & Karunochi & Verbenaceae & Leaves & $\sqrt{ }$ & & $\begin{array}{c}\text { Diarrhoea, Urticaria, } \\
\text { cellulites, abscess, } \\
\text { carbuncle, eczema. }\end{array}$ & Juice (internal) \\
\hline $\begin{array}{l}\text { Wrightia tinctoria } \\
\text { (Robx) R.Br. }\end{array}$ & Veppalai & Apocynaceae & Leaves & & $\sqrt{ }$ & $\begin{array}{l}\text { Liver disorder, asthma, } \\
\text { jaundice, piles. }\end{array}$ & $\begin{array}{l}\text { Infusion } \\
\text { (external) }\end{array}$ \\
\hline
\end{tabular}

Key: S.B - Snake bite, S.S - Scorpion sting, DB - Dog bite

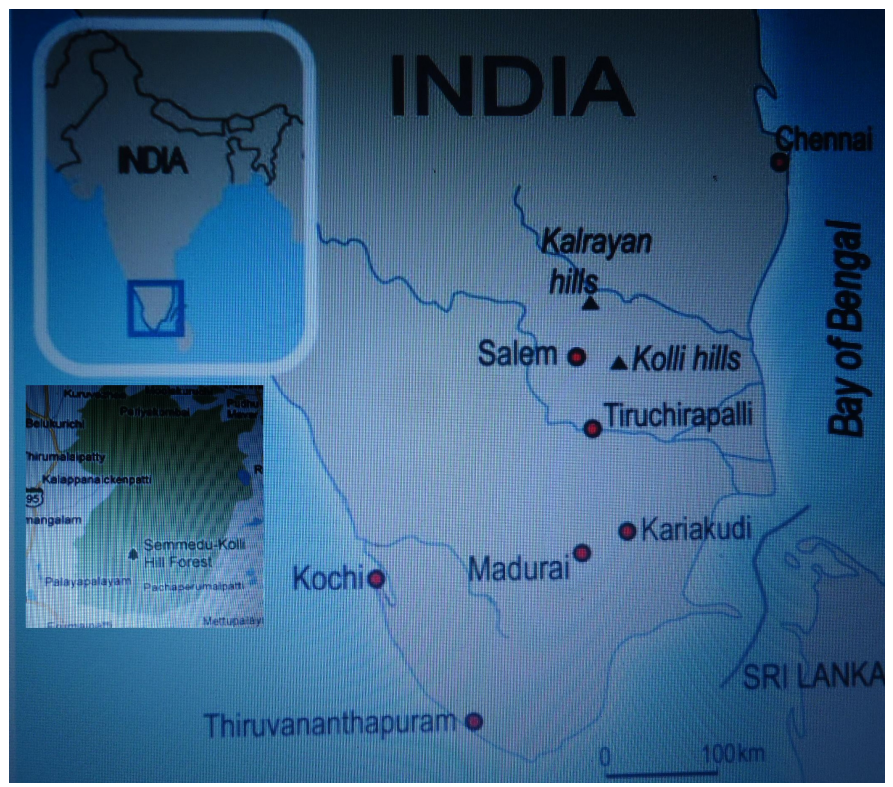

These plants are also used to cure many other diseases such as cuts, wounds, injuries, eczema, scabies, burns itches, hair growth, diarrhoea, knee pain, cold, cough, fever, typhoid, malaria, headache, sore throat, ringworm, dry skin, stomach ache, abdominal pain, intestinal worms, ulcer, gout, piles, fistula, leprosy, urinary trouble, enhance fertility, vaginal discharge, venereal diseases, nostril haemorrhage, leucorrhoea, syphilis, heart diseases, kidney stones, liver complaints, asthma, swelling, inflammation, rheumatism etc. ${ }^{14}$

The species of Rauvolfia serpentina are being used as antidote for snake bite by tribals such as Kadari, Malayali, Irular, Todar, Badagar and Kurumbar. ${ }^{15}$ The Paliyans of Tirunelveli Hills of the Western Ghats use Achyranthes aspera and Aristolochia indica to cure various poisonous bites. ${ }^{16}$ The same report was obtained from various tribals of the Kolli Hills and from Yanadis of Nallamalai Hills. ${ }^{8}$ For snake bite around 15 plants like Achyranthus aspera, Aristolochia indica, Moringa pterygosperma, Calotropis gigantia etc., belonging 11 families were reported. ${ }^{17}$ They also revealed that roots were most frequently used ( 9 species), followed by leaf extract (4 species), latex and gum with one (1) species each. Bioactive compounds and phytochemicals such as gymnemic acid may be the reason for its antidote effect against snake bite, scorpion sting and dog bite.
Ethnobotanical exploration of Chitheri Hills of Dharmapuri District reported that Anisomeles malabarica, Calotropis gigantea, Eclipta prostrata, Gloriosa superba, Moringa oleifera, Ricinus communis, Terminalia arjuna, Vernonia anthelminthica, Wrightia tinctoria and Aristolochia indica are used for scorpion sting. ${ }^{18}$

Plants such as Achyranthes aspera, Cyperus kyllingi, Datura stramonium, Moringa oleifera and Murraya koeinegii were documented as potential medicinal plants against $\operatorname{dog}$ bite. $^{19}$

\section{CONCLUSION}

The present study provides a base for enhancing scientist's attention towards consideration of ethnomedicinally important plants for snake bite, scorpion sting and dog bite. Traditional medicine practices are an important part of the primary healthcare system in the developing world. Herbal medicines are comparatively safer than synthetic drugs. Plant based traditional knowledge has become a recognized tool in search for new sources of drugs and neutraceuticals. Further studies have to be carried out to identify the phytochemicals of medicinal plants. Dissemination of the knowledge of medicinal plants and their usage would also improve the socioeconomic status of the tribes. 


\section{ACKNOWLEDGEMENT}

The authors are thankful to the tribal and local traditional healers of Kolli hills of Namakkal district for their cooperation and discussion on the subject and also revealing their valuable information in the relevant field.

\section{REFERENCES}

1. Huxley A. Green Inheritance: The World Wildlife Fund Book of India, Collins/ Harvel, London 1984.

2. Pei S J. Ethnobotanical approaches of traditional medicine studies: some experiences from Asia. Pharmacology Biology 2001; 39: 74 79. http://dx.doi.org/10.1076/phbi.39.s1.74.0005 PMid:21554174

3. Principe P. Monetizing the pharmacological benefits of plants. US Environmental Protection Agency, Washington, D.C 1991.

4. Jain S.K. Dictionary of Indian folk medicine and ethnobotany. Deep publication, New Delhi 1991.

5. WHO. General guidelines for methodologies on research and evaluation of traditional medicine. World Health Organization, Geneva 2000.

6. Ramamurthy B N. In-vitro standardization of phytochemicals importance. International seminar on Medicinal Plants/Spices, Exports-Patents. April. Chennai 2002; 133-134.

7. Martz W. Plants with a reputation against snake bite. Journal of Toxicology 1992; 30: 31-42.

8. Caius JF. Medicinal and poisonous ferns of India. Delhi, India Bombay Natural History Society. London. (Reprinted-1956) BSI, Calcutta 1935; 83: 341-361.

9. Jaya Kumar S, Arockiasamy DI and John Britto S. Forest type mapping and analysis in part of Kolli Hills in Eastern Ghats. Tropical Ecology 2002; 43 (2): 345-349.

10. Dewarakan. Additions to the flora of Kolli hills Eastern Ghats, Salem district of Tamil Nadu. Journal of Economic Taxonomical Botany 1996; 20 (3): 651.
11. Bhagya B and Sridhar KR. Ethnobiology of coastal sand dune legumes of Southwest coast of India. Indian Journal of Traditional Knowledge 2009; 8(4):611-620.

12. Matthew KM. Flora of Tamil Nadu Carnatic. Rapinat Herbarium, St. Joseph's College, Tiruchirapalli, Tamil Nadu 1983.

13. Matthew KM. An Excursion Flora of Central Tamil Nadu. Oxford and IBH Publishing Co. Pvt. Ltd, New Delhi 1991.

14. Subramoniam A Evans DA Rajasekaran, S and Pushangadan P. Effect of Trichopus zeylanicus Gaertn (active fraction) on phagocytosis by peritoneal mactrophages and humoral immune response. Indian Journal of Pharmacology 2000; 32: 221-225.

15. Alageshaboopathi $\mathrm{C}$ and Balu S. Ethnobotany of Andrographis paniculata. Acta Botanica 1997; 25: 247-250.

16. Thangadurai D. Ethnobotanical plants used as antidote for poisonous bites among the tribals South Western Ghats, India. National conference on recent trends in spices \& medicinal plant research, Culcutta, WB, India 1998; Abstract: 71.

17. Hiremath V.T and Taranath T.C. Traditional Phytotherapy for Snake bites by Tribes of Chitradurga District, Karnataka, India. Ethnobotanical Leaflets 2010; 14: 120-25.

18. Subramaniam A. A Survey of medicinal plants from Chitheri Hills in Dharmapuri District, Tamil Nadu. In: Ethnobotany and Medicinal plants of Subcontinent. 'Reprint from Journal of Economic \& Taxonomic Botany.1999; Vol. I \& II: 351-359.

19. Mohan VR, Rajesh A, Athiperumalsamy T, Sutha S. Ethnomedicinal plants of the Tirunelveli District, Tamil nadu, India. Ethnobotanical Leaflets 2008; 1-17.

\section{Cite this article as:}

Prabu M and Kumuthakalavalli R. Folk remedies of medicinal plants for Snake bites, Scorpion stings and Dog bites in Eastern ghats of Kolli hills. Int. J. Res. Ayur. Pharm. 2012; 3(5):696-700 